092

\title{
WHAT CAN WE LEARN ABOUT THE INFANT BRAIN FROM RESTING-STATE FMRI
} ACTIVITY?

\section{P. Fransson}

Dept. of Clinical Neuroscience, Karolinska Institute, Stockholm, Sweden

Recent research has shown that spontaneous activity is ubiquitous in the human brain and that it can be detected using functional magnetic resonance imaging. Intrinsically driven changes in the fMRI signal are organized in a hierarchy of so called "resting-state" networks that spans large-scale functional cortical circuits in the human brain. In my talk I will briefly describe the methodological basis and analysis used in resting-state fMRI studies and its applicability to pediatric populations. I will then focus on studies of intrinsic fMRI connectivity in the infant brain and what we can infer about the development of cortical networks from resting-state fMRI activity. Finally, I will describe the potential of using resting-state fMRI as a research tool to link differences in brain connectivity patterns to neurodevelopmental disorders. 